OPEN ACCESS

Edited by:

Max Maurin,

Université Grenoble Alpes, France

Reviewed by:

Manish K. Chourasia,

Central Drug Research Institute

(CSIR), India

Nahid Ali,

Indian Institute of Chemical Biology

(CSIR), India

*Correspondence:

Shyam Lal Mudavath

shyamla@@inst.ac.in;

shavs0502@gmail.com

Specialty section:

This article was submitted to

Clinical Microbiology,

a section of the journal

Frontiers in Cellular and Infection Microbiology

Received: 18 June 2020 Accepted: 09 September 2020 Published: 15 October 2020

Citation:

Parvez S, Yadagiri G, Karole A, Singh OP, Verma A, Sundar $S$ and Mudavath SL (2020) Recuperating

Biopharmaceutical Aspects of Amphotericin B and Paromomycin Using a Chitosan Functionalized

Nanocarrier via Oral Route for Enhanced Anti-leishmanial Activity.

Front. Cell. Infect. Microbiol.

10:570573

doi: 10.3389/fcimb.2020.570573

\section{Recuperating Biopharmaceutical Aspects of Amphotericin B and Paromomycin Using a Chitosan Functionalized Nanocarrier via Oral Route for Enhanced Anti-leishmanial Activity}

\author{
Shabi Parvez ${ }^{1}$, Ganesh Yadagiri ${ }^{1}$, Archana Karole ${ }^{1}$, Om Prakash Singh ${ }^{2}$, Anurag Verma ${ }^{3,4}$, \\ Shyam Sundar ${ }^{5}$ and Shyam Lal Mudavath ${ }^{1 *}$ \\ 1 Infectious Disease Biology Laboratory, Institute of Nano Science \& Technology, Mohali, India, ${ }^{2}$ Department of Biochemistry, \\ Institute of Science, Banaras Hindu University, Varanasi, India, ${ }^{3}$ School of Pharmaceutical Sciences, Institute of Foreign Trade \\ and Management (IFTM) University, Moradabad, India, ${ }^{4}$ Teerthanker Mahaveer College of Pharmacy, Teerthanker Mahaveer \\ University (TMU), Moradabad, India, ${ }^{5}$ Infectious Disease Research Laboratory, Department of Medicine, Institute of Medical \\ Sciences, Banaras Hindu University, Varanasi, India
}

The design and development of new pharmaceutical formulations for the existing anti-leishmanial is a new strategic alternate to improve efficacy and safety rather than new drug discovery. Herein hybrid solid lipid nanoparticles (SLN) have been engineered to direct the oral delivery of two anti-leishmanial drugs amphotericin B (AmB) and paromomycin (PM). The combinatorial nanocarriers consist of conventional SLN, antileishmanial drugs ( $\mathrm{AmB}$ and $\mathrm{PM}$ ) which have been functionalized with chitosan (Cs) grafted onto the external surface. The Cs-SLN have the mean particle size of $373.9 \pm 1.41 \mathrm{~nm}$, polydispersity index (PDI) of $0.342 \pm 0.02$ and the entrapment efficiency for AmB and PM was found to be $95.20 \pm 3.19 \%$ and $89.45 \pm 6.86 \%$, respectively. Characterization of SLN was performed by scanning electron microscopy and transmission electron microscopy. Complete internalization of the formulation was observed in Caco-2 cells. Cs-SLN has shown a controlled and slow drug release profile over a period of $72 \mathrm{~h}$ and was stable at gastrointestinal fluids, confirmed by simulated gastro-intestinal fluids study. Cs coating enhanced the mucoadhesive property of Cs-SLN. The in-vitro anti-leishmanial activity of Cs-SLN $(1 \mu \mathrm{g} / \mathrm{ml})$ has shown a maximum percentage of inhibition (92.35\%) on intra-cellular amastigote growth of $L$. donovani.

Keywords: amphotericin B, oral delivery, paromomycin, visceral leishmaniasis, solid lipid nanoparticles

\section{INTRODUCTION}

Visceral leishmaniasis (VL), also known as kala-azar is a vector-borne infectious disease caused by an obligate intracellular protozoan parasite of Leishmania species and invades the reticuloendothelial system of spleen, liver, and bone marrow (Chappuis et al., 2007; Shrestha et al., 2018; Herrera et al., 2019; Martínez and Ruiz, 2019). VL is a serious public health issue in many tropical and subtropical regions of the globe and affects poor and malnourished people 
in developing and underdeveloped countries of Brazil, East Africa, and South-East Asia. VL is the most severe form of leishmaniasis, characterized by irregular bouts of fever, anemia, weight loss, hepatosplenomegaly, and is fatal if untreated (Sundar and Singh, 2016; Herrera et al., 2019). According to World Health Organization (WHO), 50,000-90,000 new VL cases reported globally and more than $95 \%$ of new VL cases reported in 10 countries: Bangladesh, Brazil, China, Ethiopia, India, Kenya, Nepal, Somalia, South Sudan, and Sudan. ${ }^{1}$ In, Indian subcontinent, VL is highly endemic in poor rural communities of Bihar state, which contributes nearly $80 \%$ of global disease burden and threats about 200 million people (Hasker et al., 2012). Although, chemotherapy is the mainstay of therapy for VL treatment and control, the clinical application of existing anti-leishmanial agents is limited due to serious toxic adverse effects and long term intravenous drug treatment options (Yadagiri and Singh, 2018). The emergence of drugresistant parasites, HIV-VL co-infections, non-availability of effective proper vector control measures, and vaccine (s) against VL further complicated the disease control and treatment (Singh et al., 2016a; Ponte-Sucre et al., 2017). Pentavalent antimonials (sodium stibogluconate or meglumine antimoniate), the mainstay of therapy for VL have shown resistance and relapse in VL-infected patients of North Bihar, India, where VL endemicity is high (Mostafavi et al., 2019). Intravenous administration of amphotericin B for VL causes serious adverse effects including hypokalemia, myocarditis, and nephrotoxicity, which necessitates long hospital stays for close monitoring of patients and ultimately increases the cost of therapy. Aminoglycoside antibiotic, paromomycin for VL treatment causes nephrotoxicity and ototoxicity (Yadagiri and Singh, 2018). Several new VL treatment strategies have emerged during the past 10-15 years, but each has its serious own limitations. Therefore, better anti-leishmanial drug discovery is an immediate need for saving the lives of VL-infected people in endemic zones without showing any toxic adverse effects (Singh et al., 2016a).

During the last decade, nanotechnology-based drug delivery systems have been extensively used to improve the performance of drugs in treating several diseases with improved efficacy and safety. Some of the nanotechnology-based formulations have been approved by the U.S. Food and Drug Administration (FDA) for clinical application (Patra et al., 2018). Encapsulation of AmB in novel nanocarrier systems can target specific drug delivery in reticuloendothelial system of liver and spleen, where Leishmania parasites reside and replicate without showing nephrotoxicity (Gupta et al., 2012).

The need of the hour is to develop a delivery system, which annihilates the domination and inadequacy of currently available marketed formulations as well as deliver drug to macrophagespecific organs (spleen and liver). Combinational therapy is an attractive option to overcome the problems associated with the aforementioned drugs. Combinational therapy has an extensive array of advantages like shortening of the duration and lowering the cost of treatment, lowering the development of resistance. $\mathrm{PM}$ and antimonial used in short regimens were found more



effective treatment as compared to conventional drugs (MongeMaillo and López-Vélez, 2013; Singh et al., 2016b). Kun Shi et al. (2019) reported combinational therapy of gemcitabine and cis-platinum via thermo-sensitive copolymer micelles to target pancreatic cancer (Shi et al., 2019). Trinconi et al. (2014) reported the effective role of combination therapy of tamoxifen and $\mathrm{AmB}$ for cutaneous leishmaniasis gave good clinical as well as parasitological response (Trinconi et al., 2014). Hence, combinational therapy has ascertained to be more effective as compared to single-drug therapies.

The oral route is the most convenient and safest route along with the highest patient compliance, low cost, and lesser complication in comparison to other routes of administrations. Poor solubility and poor permeability of drugs are needed to be considered for the enhancement of oral bioavailability. To overcome the obstacle allied with oral drug delivery systems, nanosized or nanoscale (NP) particles could be considered as an excellent alternative to conventional drug delivery systems and generally used to augment the oral bioavailability of drugs. Solid lipid nanoparticles (SLN) have attracted the attention of our research group to develop a formulation with enhanced oral bioavailability of antileishmanial drugs.

SLN is a colloidal carrier (Vivek et al., 2007) which is a classically sphere-shaped average particle size found in the range of 50-500 nm (Chavan et al., 2013). SLN are composed of solid lipid core matrix which can solubilize lipophilic drug. The lipid core is become stable by surfactant (Vivek et al., 2007). SLN holds various advantages like high drug payload, long term stability, controlled release profile. Due to the unique characteristics of SLN, it could be used for delivery of both hydrophilic (PM) and hydrophobic $(\mathrm{AmB})$ drugs, depending on the type of lipid, surfactant, and method of preparation (Mendonça et al., 2020).

Chitosan (Cs) is a polycationic naturally occurring bio-degradable, non-toxic, non-allergenic bio-polysaccharide derived from chitin which is abundant in nature (Jain et al., 2018). Cs is studied as the most efficient material for impending use on account of its exemplary bio-degradability, biocompatibility, non-toxicity, antimicrobial activity, and is cost-effective. Cs exhibits escalating mucoadhesive properties that expedite and enhance the transport capacity of drugloaded nanoparticles, their absorption across the GI tract, and eventually improve the drug bio-availability when administered orally (Singh et al., 2016b; Min et al., 2018). Surface modification of nanoformulation could have been used for the customization of therapeutic efficiency as well as the biodistribution profile (Jain et al., 2015).

In this study, Cs-coated SLN containing AmB and PM were developed and characterized to be tested as oral carriers for AmB and PM. NPs were assessed considering their physicochemical properties, stability on simulated GI fluids, and cytotoxicity over J774A.1 cells, cellular uptake studies, mucoadhesive property, and anti-leishmanial activity.

\section{MATERIALS AND METHODS}

\section{Materials}

Chitosan (low molecular weight), 4', 6-diamidino-2phenylindole dihydrochloride (DAPI), fluorescein isothiocyanate 
(FITC), 3-(4, 5-dimethylthiazol-2-yl)-2,5-diphenyltetrazolium bromide (MTT) were purchased from Sigma-Aldrich (USA). AmB, glycerol monostearate (GMS), polyvinyl alcohol (PVA), Tween 80, polyethylene glycol 400 (PEG 400), 4\% paraformaldehyde and cellulose dialysis tube $(12 \mathrm{kDa})$ were procured from HiMedia Laboratories (India). RPMI 1640, heatinactivated fetal bovine serum (HI-FBS), penicillin-streptomycin, rhodamine-phalloidin, trypsin $0.25 \%$, glass-bottom dishes and phosphate buffer saline (PBS; $\mathrm{pH}$ 7.4) were purchased from ThermoFisher Scientific (India). Sodium taurocholate, paromomycin (PM) were obtained from SRL (India).

\section{Parasite and Cell Line}

Leishmania donovani (LEM 138) parasites were cultured in M199 medium supplemented with antibiotics $(100 \mathrm{U} / \mathrm{ml}$ penicillin, $100 \mu \mathrm{g} / \mathrm{ml}$ streptomycin) and $10 \%$ heat-inactivated fetal bovine serum (HI-FBS), maintained at $26^{\circ} \mathrm{C}$ BOD incubator. J774A.1 macrophage cells were cultured in RPMI-1640 supplemented with penicillin $(100 \mathrm{U} / \mathrm{ml})$ and streptomycin $(100 \mu \mathrm{g} / \mathrm{ml})$ and HI-FBS (10\%), maintained at $37^{\circ} \mathrm{C}$ and $5 \% \mathrm{CO}_{2}$ incubator.

\section{Fabrication of Unmodified/Surface-Modified Drug-Loaded Solid Lipid Nanoparticle}

AmB and PM loaded SLN (DSLN) were prepared by the emulsion solvent evaporation method (Liu et al., 2011). Briefly, GMS $(150 \mathrm{mg}), \mathrm{AmB}(50 \mathrm{mg})$ were dispersed in $5 \mathrm{ml}$ of ethanol and soy lecithin $(40 \mathrm{mg})$, heated to $60^{\circ} \mathrm{C}$ to compose the organic phase. The aqueous phase was formulated simultaneously by the addition of PVA $(0.5 \% \mathrm{w} / \mathrm{v})$, PEG $400(1.5 \% \mathrm{w} / \mathrm{v})$, and PM $(20 \mathrm{mg})$, in double-distilled water $(20 \mathrm{ml})$ maintained at $60^{\circ} \mathrm{C}$. The organic phase was added drop by drop into aqueous phase with constant stirring at $1000 \mathrm{rpm}$, leading to the formation of an emulsion. The emulsion was then released to another aqueous phase; 1\% Tween $80(\mathrm{w} / \mathrm{v})$ and 1\% of PEG $400(\mathrm{w} / \mathrm{v})$ and stirred continuously at $4{ }^{\circ} \mathrm{C}$ allowing the solidification of SLN. The resulting suspension was centrifuged at 11,000 rpm for 30 min (Avanti JXN-30 Beckman Coulter US) to collect the pellet, which was washed thrice with Milli-Q water. Mannitol (2\% w/v) was added as a cryoprotectant and the resulting dispersion was freeze-dried (FDUT-12003, Republic of Korea) to get the dried DSLN. Formulated DSLN coated with Cs (Cs-SLN) were prepared by incubating the freeze-dried DSLN with chitosan $(0.1 \% \mathrm{w} / \mathrm{v})$ solution for $2 \mathrm{~h}$ at room temperature (Channarong et al., 2011; Guo et al., 2016). Plain SLN (PSLN) was prepared by a similar method without the drugs. FITC labeled Cs-SLN were prepared by adding FITC in the organic phase in the preparation methodology as described above.

\section{Encapsulation Efficiency and Drug Loading} Encapsulation efficiency and drug loading was determined by using a previously reported indirect method. Fmoc-PM complex was synthesized by solution-phase process for the determination of PM, Fmoc- $\mathrm{Cl}(4 \mathrm{mM})$ was added to acetonitrile and subsequently add to PM $(0.5 \mathrm{mM})$ in borate buffer $(\mathrm{pH} 8.1)$ and stirred in completely dark conditions for few minutes. Ethyl acetate was used for extraction and the complex obtained was further used for the development of formulations.

After the preparation of DSLN, the supernatant was collected by centrifugation at $11,000 \mathrm{rpm}$ for $30 \mathrm{~min}$ and it was used to measure the amount of Fmoc-PM complex at $315 \mathrm{~nm}$ by using fluorescence spectroscopy (F-4600 spectrophotometer, Hitachi, Japan) (Kumar and Bose, 2016) and AmB was determined using UV-Visible spectrophotometer (Shimadzu UV-2600) at $405 \mathrm{~nm}$.

The entrapment efficiency and drug loading was determined by the following formulae.

$$
\begin{aligned}
& \text { Entrapment efficiency }(\% \mathrm{EE})=\mathrm{W}_{\mathrm{t}}-\mathrm{W}_{\mathrm{s}} / \mathrm{W}_{\mathrm{t}} * 100 \\
& \text { Drug loading }(\% \mathrm{DL})=\mathrm{W}_{\mathrm{t}}-\mathrm{W}_{\mathrm{s}} / \mathrm{W}_{\mathrm{l}}+\mathrm{W}_{\mathrm{t}}-\mathrm{W}_{\mathrm{s}} * 100
\end{aligned}
$$

where, $\mathrm{W}_{1}$ is the amount of lipid, $\mathrm{W}_{\mathrm{t}}$ is the total amount of drug used, $W_{s}$ the drug remaining in the supernatant.

\section{Characterization}

\section{Particle Size and Zeta Potential}

Particle size, polydispersity index (PDI), and zeta potential of freshly prepared Cs-SLN were determined by using photon correlation spectroscopy (Zetasizer Nano ZSP; Model-ZEN5600, Malvern Instruments Ltd. UK) at $25^{\circ} \mathrm{C}$. Concisely, Cs-SLN were sufficiently diluted with Milli-Q water (1:100) and placed in disposable cuvettes for the measurement of particle size and in disposable folded capillary cells for determination of zeta potential.

\section{Morphology}

Morphology of Cs-SLN was observed using scanning electron microscopy (SEM) and transmission electron microscopy (TEM). The Cs-SLN dispersion was drop-casted and then sputter-coated with gold under vacuum (JEC- 300 FC) and analyzed for SEM. For the TEM (JEOL 2100) analysis, the dispersion was drop cast onto 300 mesh carbon coated-copper grid and the surplus amount was taken off with filter paper and negatively stained with $2 \% \mathrm{w} / \mathrm{v}$ phosphotungstic acid (PTA) for contrast enhancements. Further, the samples were kept overnight for vaccum drying. TEM images were captured at an accelerating voltage of $200 \mathrm{kV}$, using Gatan camera software.

\section{FTIR Spectroscopy}

Fourier transform infrared spectroscopy (FTIR) was performed to ascertain any chemical interactions present between GMS, AmB, PM, Cs, and Cs-SLN using Bruker vertex 70v spectrophotometer and were scanned at $400-4,000 \mathrm{~cm}^{-1}$ for at the resolution of $4 \mathrm{~cm}^{-1}$ and 64 scans performed for all samples.

\section{Powder X-ray Diffractometer (PXRD)}

X-ray diffraction (XRD) patterns of AmB, PM, Cs, and Cs-SLN were obtained by using Bruker, D8 advance X-ray diffractometer outfitted with a Ni-filtered $\mathrm{Cu} \mathrm{K} \alpha$-radiation, $\lambda=1.541 \AA$ (voltage $40 \mathrm{kV}$; current $30 \mathrm{~mA}$ ). The diffraction pattern was recorded over a $2 \theta$ range of $10-70^{\circ}$ at a scanning rate of $5^{\circ}$ per min. 


\section{Cellular Uptake}

Confocal microscopy was used to study the internalization of FITC labeled Cs-SLN in Caco-2 cells to evaluate the oral uptake efficiency of Cs-SLN. Briefly, Caco-2 cells were seeded at an initial density of 10,000 cells per well in glass-bottom dishes in Dulbecco's modified Eagle's medium (DMEM) with $20 \%$ fetal bovine serum (FBS), $100 \mu \mathrm{g} / \mathrm{ml}$ streptomycin and $100 \mathrm{IU} / \mathrm{ml}$ penicillin, and placed in an incubator at $37^{\circ} \mathrm{C}$ for $48 \mathrm{~h}$ allowing the cells to adhere. The cells were treated with FITC labeled Cs-SLN for 2 and $6 \mathrm{~h}$. The cells were washed thrice with 1X PBS $(\mathrm{pH} \mathrm{7.4)}$ and fixed with $4 \%$ paraformaldehyde for $20 \mathrm{~min}$. The cells were washed thrice with PBS ( $\mathrm{pH} 7.4$ ) and stained with DAPI ( $1 \mu \mathrm{g} / \mathrm{ml})$ for 4-5 min. Rhodamine-phalloidin was used to stain the cell membrane for $30 \mathrm{~min}$. The cells were washed and images were acquired using a confocal laser scanning microscope (CLSM, LSM 880 NLO, Carl Zeiss, Germany).

\section{In vitro Cytotoxicity Study}

MTT assay was used to evaluate the cytotoxicity of SLN on J774A. 1 macrophage cells. In brief, $5 \times 10^{4}$ cells/well were seeded into 96 well plates and were kept at $37^{\circ} \mathrm{C}$ and $5 \% \mathrm{CO}_{2}$ environment, to adhere to cells for $24 \mathrm{~h}$. The treatment to the cells was given with varying concentrations $(3.12 \mu \mathrm{g} / \mathrm{ml}, 6.25 \mu \mathrm{g} / \mathrm{ml}$, $12.50 \mu \mathrm{g} / \mathrm{ml}, 25 \mu \mathrm{g} / \mathrm{ml}, 50 \mu \mathrm{g} / \mathrm{ml}$ ) of PSLN, Cs-SLN, and free drugs (AmB and PM) as well as the untreated cells were used as control. At a predetermined time, MTT reagent $(20 \mu \mathrm{l}$ of 5 $\mathrm{mg} / \mathrm{ml}$ stock solution) was added followed by incubation for $4 \mathrm{~h}$, under $5 \% \mathrm{CO}_{2}$ environment, and at $37^{\circ} \mathrm{C}$. After the removal of the medium, DMSO $(100 \mu \mathrm{l})$ was added to dissolve formazan crystals. Cell viability was evaluated by was measuring the optical density (OD) of solutions by using a microplate reader (Infinite $200 \mathrm{PRO}$ microplate plate reader) at $570 \mathrm{~nm}$ as well as $630 \mathrm{~nm}$ used as a reference wavelength (Khatik et al., 2014). Cell viability was calculated by applying the formula:

$$
\text { Cell viability }=\mathrm{Abs}_{\mathrm{s}} / \mathrm{Abs}_{\mathrm{c}} * 100
$$

where, $\mathrm{Abs}_{\mathrm{s}}$ is the absorbance of samples, and $\mathrm{Abs}_{\mathrm{c}}$ is the absorbance of control.

\section{In vitro Release Study}

Dialysis membrane method was used to evaluate the in vitro drug release study. The dialysis membrane $(12 \mathrm{kDa})$ was soaked in Milli-Q water overnight and formulations were placed in dialysis membrane closed at both the ends with the clamps, which was then immersed in $250 \mathrm{ml}$ phosphate buffer saline $(\mathrm{pH} 7.4)$ along with $1 \%$ Tween 80 and agitated at $150 \mathrm{rpm}, 37 \pm 1^{\circ} \mathrm{C}$ for $72 \mathrm{~h}$. Aliquots were taken out at regular time intervals and replaced with fresh buffer to maintain the sink conditions. Samples were analyzed using UV-VIS spectroscopy (Shimadzu UV-2600) at $413 \mathrm{~nm}$ and by using fluorescence spectroscopy (Infinite $200 \mathrm{PRO}$ microplate plate reader) for detection of PM at $312 \mathrm{~nm}$.

\section{In vitro Simulated Gastrointestinal Fluid Stability Study}

To evaluate the in vitro stability while passing through the GIT tract, Cs-SLN were placed in hydroxypropyl methylcellulose
(HPMC) capsule and kept in individual dissolution baskets containing $500 \mathrm{ml}$ medium (simulated gastric fluid (SGF; $\mathrm{pH}$ 1.6) and simulated intestinal fluids (SIF; $\mathrm{pH}$ 6.5) maintained at $37 \pm 0.5^{\circ} \mathrm{C}$ under continuous stirring at $50 \mathrm{rpm}$. The SGF was prepared by adding sodium chloride $(34.2 \mathrm{mM})$, lecithin $(20 \mu \mathrm{M})$, sodium taurocholate $(80 \mu \mathrm{M})$, pepsin $(0.1$ $\mathrm{mg} / \mathrm{ml}$ ), conc. $\mathrm{HCl}$ (q.s. $\mathrm{pH} 1.2$ ) in $500 \mathrm{ml}$ deionised water and SIF was prepared by adding lecithin $(0.75 \mathrm{mM})$, sodium taurocholate $(3 \mathrm{mM})$, sodium chloride $(6.18 \mathrm{gm})$, sodium dihydrogen phosphate $(3.43 \mathrm{gm})$, and sodium hydroxide (q.s. $\mathrm{pH}$ $6.5)$ in $500 \mathrm{ml}$ deionised water. Samples were taken out regularly and quantified using UV-VIS spectroscopy (Shimadzu UV-2600) at $408 \mathrm{~nm}$ (Klein, 2010).

\section{Mucoadhesive Property}

Freshly prepared Cs-SLN were evaluated using the main glycoprotein in mucus i.e., mucin at different concentrations $(0.1,0.25$, and $0.5 \% \mathrm{w} / \mathrm{v})$, in $0.02 \mathrm{M}$ phosphate buffer $\mathrm{pH} 6.8$. Cs-SLN dispersion was incubated with mucin solutions for $30 \mathrm{~min}$ in a shaker at $70 \mathrm{rpm}, 37^{\circ} \mathrm{C}$. Particle size, PDI, Zeta potential were recorded before and after the incubation period (Pauluk et al., 2019).

\section{In vitro Anti-leishmanial Activity of Cs-SLN Against $L$. donovani Amastigotes}

In vitro anti-leishmanial activity of Cs-SLN, AmBisome, and AmB were tested against intracellular amastigotes of Leishmania parasite. Briefly, macrophage cells (J774A.1) $\left(2.5 \times 10^{5}\right.$ cells $\left./ \mathrm{ml}\right)$ were resuspended in complete RPMI-1640 and seeded in eight well Lab Teck tissue culture slides (Nunc, USA) and incubated at $37^{\circ} \mathrm{C}$ and $5 \% \quad \mathrm{CO}_{2}$ environment for $2 \mathrm{~h}$ for macrophage adherence. The adherent macrophages were washed ( $\times 3)$ with pre-warmed incomplete RPMI-1640 and infected with metacyclic promastigotes in $1: 10$ ratio and incubated at $37^{\circ} \mathrm{C}$ and $5 \% \mathrm{CO}_{2}$ environment for $12 \mathrm{~h}$. Non-phagocytized promastigotes were discarded by a simple exchange of medium and the infected macrophages were incubated with and without having the test and reference drugs (Cs-SLN, AmBisome, and AmB) in different concentrations $(0.1-1 \mu \mathrm{g} / \mathrm{ml})$ in complete RPMI-1640 at $37^{\circ} \mathrm{C}$ and $5 \% \mathrm{CO}_{2}$-air atmosphere for $72 \mathrm{~h}$. The infected macrophages were washed with PBS and stained with Wright's stain to assess the intracellular amastigote growth and intracellular amastigotes were monitored by counting at least 100 cells per slide under the oil immersion lens microscope (100×) (Nahar et al., 2009).

Percentage inhibition of amastigote replication was calculated by the following formula:

PI $=100-(\mathrm{AT} / \mathrm{AC}) \times 100$
PI: percentage inhibition of amastigote multiplication,
AT: actual number of amastigotes in treated
samples/100 macrophages;
AC: actual number of amastigotes in control
samples/100 macrophages.

Statistical Analysis Origin (version 8.6) and GraphPad Prism (version 8.0.2) software were used to analyze the data of various groups of experiments. Graphs represent data from an average of 
3 experiments. ${ }^{* * * *} P$-value $\leq 0.0001,{ }^{* * *} P$-value $\leq 0.001,{ }^{* *} P$ value $\leq 0.01$, and ${ }^{*} P$-value $\leq 0.05$ two-way ANOVA, Tukey's multiple comparisons test. The data from individual groups were presented as the mean $\pm \mathrm{SD}$.

\section{RESULTS AND DISCUSSION \\ Fabrication of Nanoparticles and Characterization}

SLN were successfully developed by using the ESE method. Formulation and process variables were optimized based on mean particle size and PDI. Optimized formulation has shown a small mean particle size of $37.6 \pm 0.38 \mathrm{~nm}$ with a PDI of $0.31 \pm$
0.03 (Table 1). Nanoparticles with small size and spherical shape can be prepared by ESE (Cavallaro et al., 2015). The mean particle size of DSLN were considerably increased to $148 \pm 3.60 \mathrm{~nm}$ with PDI $0.308 \pm 0.05$ and zeta potential of $+1.140 \pm 0.01 \mathrm{mV}$ as compared to PSLN $(37.6 \pm 0.38 \mathrm{~nm})$. Surface modifications of formulation with $\mathrm{Cs}$, further increases the mean particle size to $373.9 \pm 1.41 \mathrm{~nm}$, respectively (Figures $\mathbf{1 A , B}$ ). Structure as well as the morphology of Cs-SLN were verified by SEM and TEM. SLN are uniformly distributed in shape and size for both the modifications by SEM analysis $307-410 \mathrm{~nm}$ (Figure 1C). Surface modified formulations have shown spherical shape with a smooth surface in TEM measurements (Figure 1D). Entrapment efficiency (\%) was found to be $95.20 \pm 3.19 \%$ and $89.45 \pm 6.86 \%$ for $\mathrm{AmB}$ and $\mathrm{PM}$, respectively. AmB can easily encapsulated

TABLE 1 | Particle size and zeta potential of surface-modified dual drug-loaded formulation. Results are presented as mean \pm standard deviation ( $n=3$ ).

\begin{tabular}{|c|c|c|c|c|c|c|c|}
\hline \multirow[t]{2}{*}{ Formulations } & \multirow{2}{*}{$\begin{array}{c}\text { Mean particle } \\
\text { size }(\mathrm{nm})\end{array}$} & \multirow[t]{2}{*}{ PDI } & \multirow{2}{*}{$\begin{array}{c}\text { Zeta } \\
\text { potential } \\
(\mathrm{mV})\end{array}$} & \multicolumn{2}{|c|}{ AmB } & \multicolumn{2}{|c|}{ PM } \\
\hline & & & & $\%$ EE & $\%$ DL & $\%$ EE & $\% \mathrm{DL}$ \\
\hline PSLN & $37.6 \pm 0.38$ & $0.31 \pm 0.03$ & $-7 \pm 0.316$ & NA & NA & NA & NA \\
\hline DSLN & $148 \pm 3.60$ & $0.308 \pm 0.05$ & $1.14 \pm 0.01$ & $95.49 \pm 2.99$ & $23.00 \pm 2.79$ & $89.97 \pm 4.06$ & $10.5 \pm 1.98$ \\
\hline Cs-SLN & $373.9 \pm 1.41$ & $0.342 \pm 0.02$ & $18 \pm 0.07$ & $95.20 \pm 3.19$ & $22.89 \pm 2.67$ & $89.45 \pm 6.86$ & $10.4 \pm 1.83$ \\
\hline
\end{tabular}



(b)

Zeta Foential Dstribution


FIGURE 1 | (A) Mean particle size (nm) of Cs-SLN. (B) Zeta potential (mV) of Cs-SLN. (C) SEM image of Cs-SLN (scale bar, $5 \mu$ m). (D) TEM image of Cs-SLN with $200 \mathrm{~nm}$ magnification. 
within SLN in the lipid matrix due to its lipophilic nature. Lipid-based formulation of $\mathrm{AmB}$ has low aqueous solubility (Battaglia et al., 2010).

\section{FTIR Study}

The characteristic peaks in FTIR spectra of GMS are present at 2,915 and $2,852 \mathrm{~cm}^{-1}$ (C-H stretch in the $-\mathrm{CH}_{2}$ groups), at $1,733 \mathrm{~cm}^{-1}\left(\mathrm{C}=\mathrm{O}\right.$ stretching, fatty acid ester), at $1,108 \mathrm{~cm}^{-1}(\mathrm{C}-$ $\mathrm{O}-\mathrm{C}$ stretching), $3,300 \mathrm{~cm}^{-1}$ (O-H stretching, glycerol moiety), $1,250 \mathrm{~cm}^{-1}$ (C-O stretch), 1,457 and $718 \mathrm{~cm}^{-1}$ (C-H stretching). The FTIR spectrum showed characteristic peaks of PM at 1,534 $\mathrm{cm}^{-1}\left(\mathrm{CH}_{2}\right.$ bending) at $1,626 \mathrm{~cm}^{-1}(\mathrm{~N}-\mathrm{H}$ bending coupled with $\mathrm{C}-\mathrm{N}$ stretch), and at $1,019 \mathrm{~cm}^{-1}$ (C-O-C) stretch are present in spectra of PM (Khan and Kumar, 2011). The FTIR spectra of $\mathrm{AmB}$ showed characteristic peaks such as $3,368 \mathrm{~cm}^{-1}(-\mathrm{OH}$ stretch strongly $\mathrm{H}$ - bond), $3,011 \mathrm{~cm}^{-1}$ (C-H stretch, polyene), $1,553 \mathrm{~cm}^{-1}$ (N-H in-plane), and $1,687 \mathrm{~cm}^{-1}$ ( $\mathrm{C}=\mathrm{O}$ stretching), $1,310 \mathrm{~cm}^{-1}$ (C-O stretching) shown in Figure 2A (Singh et al., 2016b). In the FTIR spectrum of Cs-SLN peaks corresponding to AmB and PM were found at $3,348 \mathrm{~cm}^{-1}, 3,011 \mathrm{~cm}^{-1}, 1,681 \mathrm{~cm}^{-1}$, $1,552 \mathrm{~cm}^{-1}$ and $1,636 \mathrm{~cm}^{-1}, 1,534 \mathrm{~cm}^{-1}$, respectively. There was no new peak found in the spectra of Cs-SLN which indicates the presence of drugs in dissolved form in the lipid matrix and no interaction was observed between the drugs and lipid matrix (Ghadiri et al., 2011; Butani et al., 2016).

In the IR spectra of chitosan (Figure $2 \mathrm{~A}) 3,367 \mathrm{~cm}^{-1}(\mathrm{O}-\mathrm{H}$ stretching overlapping the $\mathrm{N}-\mathrm{H}$ stretching), 2,867 $\mathrm{cm}^{-1}$ (C$\mathrm{H}$ stretching), $1,637 \mathrm{~cm}^{-1}$ (N-H bending), 1,585 $\mathrm{cm}^{-1}(\mathrm{~N}-\mathrm{H}$ stretching) $1,435-1,386 \mathrm{~cm}^{-1}$ (asymmetrical C-H bending of the $\mathrm{CH}_{2}$ group) and $1,027 \mathrm{~cm}^{-1}$ (C-N stretching). In the IR spectra of Cs-SLN the characteristic peaks of chitosan are present. Some peaks are shifted and broadened due to overlapping of groups and formation of nanoformulation.

\section{Powdered X-Ray Diffraction Study (PXRD)}

PXRD was performed to determine the physical state of lipid, AmB, and PM. Lipid and AmB showed a compact and characteristic diffraction pattern. X-ray diffractogram of $\mathrm{AmB}$ has exhibited a sharp peak at $2 \theta$ scattered angle of $21.39,14.1$, and $21.78^{\circ}$ indicating its crystallinity but PM was found to be amorphous in nature. Cs-SLN showed two peaks at 24.8 and $30.6^{\circ}$ but the pattern was showing shifted, broadened, and weaker peak as compared to GMS, which was partially recrystallized and transformed to less ordered in SLN formulation. Drug peaks were also absent due to complete entrapment of drug in the lipid matrix. The less ordered and amorphous nature would be contributing to higher drug loading. Diffraction peaks broadening was related to particle sizes as the broadening of Bragg's peaks indicates the formation of nanoparticles (Figure 2B) (Kumar et al., 2016).

\section{Cellular Uptake Studies}

Confocal laser scanning microscopy studies were performed to FITC-tagged Cs-SLN to assess drug internalization into Caco- 2 cells. It was observed that the FITC-tagged Cs-SLN were internalized by Caco- 2 cells in the time-dependent way as well as no toxic response was seen because there was
A



B



FIGURE 2 | (A) FTIR Spectra of GMS, PM, AmB, Cs, Cs-SLN. (B) XRD Spectra of GMS, AmB, PM, Cs, Cs-SLN. 
no change observed in the morphology of the Caco-2 cells (Figure 3). Chitosan could be used for macrophage targeting as it causes the activation of macrophages and it enhances uptake of NPs by kupffer cells (passive targeting). It was previously reported that Curcumin SLN has revealed augmented uptake in Caco2/HT-29-MTX cell monolayer up to $2 \mathrm{~h}$ (Guri et al., 2013). The SLN uptake efficiency can be enhanced due to surface coating (Costa et al., 2018). C6-labeled SLN and NLCs showed enhanced internalization in a time-dependent manner on HaCaT and CCC-ESF cells (Guo et al., 2015). It was observed during initial experiments that the morphology of the cells was not changed as well as normal doubling time (i.e., $48 \mathrm{~h}$ ) even in the presence of Cs-SLN. The cells were healthy and no sign of apoptosis was observed (Vijayakumar et al., 2017). Schipper et al. (1997) reported that chitosan acts as an absorption enhancer for the poorly absorbable drugs (Schipper et al., 1997).

The higher cellular uptake could be due to the cationic surface charge of nanoparticles or might be due to the efficient binding of Cs-SLN to the cell membrane (Ma and Lim, 2003; Wang et al., 2016).
Chitosan is being accepted as a fascinating material for oral route delivery, which could be used as a permeation enhancer, mucoadhesion, and P-gp inhibition (Liu et al., 2017).

\section{In vitro Cytotoxicity Study}

The cell viability is a common assay for the assessment of cytotoxicity of Cs-SLN, PSLN, PM, and AmB in macrophage cell lines (J774A.1) by MTT assay. The cell cytotoxicity of Cs-SLN, PSLN, PM were found to be less toxic when compared to free $\mathrm{AmB}$, at all equivalent concentrations (Figure 4). Cell cytotoxicity study confirmed the safety of excipient used for the development of nanoparticles as the PSLN were showing more than $80 \%$ viability at all concentrations. $\%$ Cell viability reduction was found to be dose-dependent as it was observed that cell viability was lowest at $50 \mu \mathrm{g} / \mathrm{ml}$ of Cs-SLN. This was reflected from the controlled drug release pattern of $\mathrm{AmB}$, which was showing $32.6 \%$ of drug release within $24 \mathrm{~h}$. The encapsulation of AmB in SLN could be the reason for reduced toxicity and an additional surface coating with biocompatible polymers did not affect the cell viability (Ching et al., 1983). Ling et al. reported that $\mathrm{AmB}$ slow release from the lipid matrix could be the reason



FIGURE 3 | Confocal microscopy images of Caco-2 cells after $6 \mathrm{~h}$ incubation at $37^{\circ} \mathrm{C}$ with FITC-tagged Cs-SLN. FITC, fluorescein isothiocyanate; hrs, hours. 
behind lower higher cell viability of Cs coated formulation (Ling et al., 2019). SLN formulations are granted as low- or non-toxic because they are produced using biodegradable compounds, which are commonly used in pharmaceuticals and cosmetics, also called GRAS (Generally Regarded As Safe) and free from the risk of acute and chronic toxicity (Bagde et al., 2019).

\section{In vitro Drug Release Study}

Drug release profile showed a biphasic pattern for both the drugs released from Cs-SLN (Figure 5A). Within an initial $6 \mathrm{~h}$, $27.6 \% \mathrm{AmB}$, and $34.4 \% \mathrm{PM}$ was found to be released due to the burst release. Being hydrophobic in nature AmB was present in the lipid core and remains inside till lipid erosion or lipid


FIGURE 5 | (A) \% Cumulative drug release study of PM and AmB for Cs-SLN. (B) In vitro simulated gastric fluid drug release study of Cs-SLN. Results are presented as mean \pm standard deviation $(n=3)$. 
degradation process gets initiated, apart from that PM, being hydrophilic presents in the outer surfactant layer. The presence of $\mathrm{PM}$ on the outer layer or a little erosion of chitosan coating, might be one or both could be the reason, which leads to the higher burst release effects. Methazolamide-chitosan-SLN dispersion exhibited a biphasic release pattern, with an initial burst release about $50 \%$ within the first two hours followed by a sustained release in the following $6 \mathrm{~h}$ (Pozo-rodríguez et al., 2009). As observed during the drug release study \% CDR (33.9\%) for AmB and $37.21 \%$ for PM up to $72 \mathrm{~h}$, revealed controlled and slow drug release from Cs-SLN. SLN has before now acknowledged as the controlled release drug carrier. It was previously reported that sustained drug release could be accomplished when the drug is uniformly dispersed in the lipid matrix (Almeida, 2017). Percentage drug release of carbamazepine (CBZ) was observed for a different formulation of SLN and $\sim 66.7 \%$ of drug was released in $24 \mathrm{~h}$. CBZ encapsulated in chitosan SLN showed a controlled drug release (Nair et al., 2012). Surface modification or assembly of biological polymer coating could be employed to develop sustained release characteristics of SLN. Cisplatin-loaded chitosan-coated SLN showed a significantly higher apoptosis in cancer cells, which is attributed to the increased internalization of nanocarriers and the controlled release of anticancer drugs in the intracellular environment (Wang et al., 2016).

\section{In vitro Simulated Gastric and Intestinal Fluid Study}

To monitor the stability of SLN, it is very important to study the effects of gastro-intestinal fluids on the developed formulations. $\mathrm{AmB}$ release was found to be negligible in SGF ( $\mathrm{pH} 1.6)$ and SIF ( $\mathrm{pH}$ 6.5) as shown in Figure 5B. The chitosan coating prevents the premature RVT release when compared to uncoated nanoparticles (Pauluk et al., 2019). Luo and co-workers reported that the presence of chitosan coating protects the constituent drug from the effect of gastric enzymes (Luo et al., 2011).
Mucoadhesive properties of chitosan enhance the interaction with the membrane as well as the absorption of compounds by the gastrointestinal tract (Farris et al., 2017).

\section{Mucoadhesive Property}

Mucin is negatively charged owing to sialic acid and CsSLN possessed positive charge before incubating with different mucin solutions because of the presence of an amine group. Further, a drop in the value of zeta potential was observed, which moved toward more negative, revealed the presence of interaction between the amine group of chitosan and mucin, reflecting mucoadhesive property of chitosan (Figures 6A,B) (Ling et al., 2019).

\section{In vitro Anti-leishmanial Activity of Cs-SLN Against $L$. donovani-Infected J774A.1 Macrophages}

Anti-leishmanial activity of Cs-SLN, AmBisome, and AmB were evaluated against $L$. donovani-infected macrophages, in vitro. All samples were stained and intra-cellular amastigotes were enumerated. Cs-SLN $(1 \mu \mathrm{g} / \mathrm{ml})$ have significantly $(P<0.01)$ diminished the intra-cellular amastigotes compared to free $\mathrm{AmB}$ (Figure 7C). The $\mathrm{IC}_{50}$ values of Cs-SLN, AmBisome, and AmB were observed to be $0.018422 \pm 0.005928 \mu \mathrm{g} / \mathrm{ml}, 0.193894 \pm$ $0.015368 \mu \mathrm{g} / \mathrm{ml}$, and $0.316039 \pm 0.026423 \mu \mathrm{g} / \mathrm{ml}$, respectively (Figure 7B). The IC50 value of Cs-SLN, was significantly lower than AmBisome $(P<0.05)$ and $\mathrm{AmB}(P<0.01)$. The IC50 value of Cs-SLN was 17.5- and 10.7-fold lower than the IC50 value of free AmB and Ambisome, respectively. The enhanced anti-leishmanial activity in macrophages is due to surface modification with Cs, which could be accountable for enhanced macrophage internalization. Cs can withstand the lysosomal $\mathrm{pH}$ owing to its acid resistive nature. Cs is also known to target macrophages via glucosamine-like receptors displayed on the surface of kupffer cells, the resident liver macrophages.
A

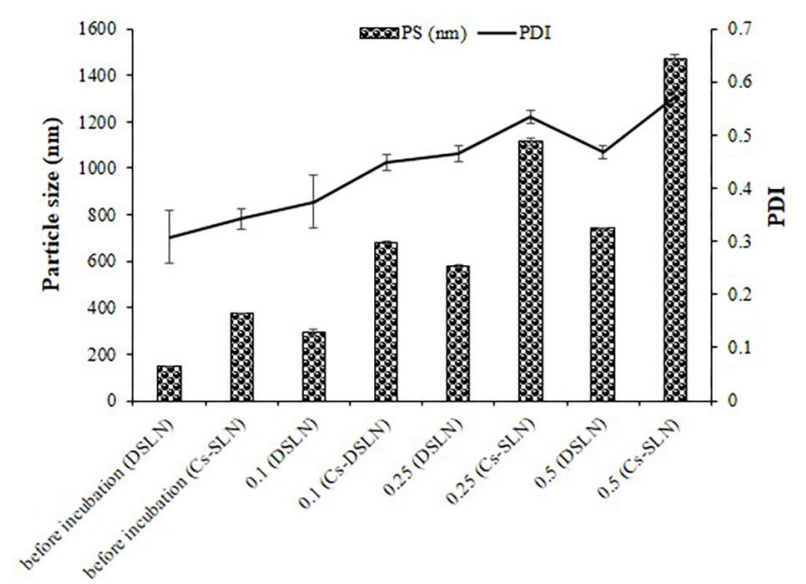

B



FIGURE 6 | (A) Mean particle size, PDI of DSLN, Cs-SLN in the absence and presence of mucin. (B) Zeta potential of DSLN, Cs-SLN in the absence and presence of mucin. Results are presented as mean \pm standard deviation $(n=3)$. 
A



B



C



Infected Control

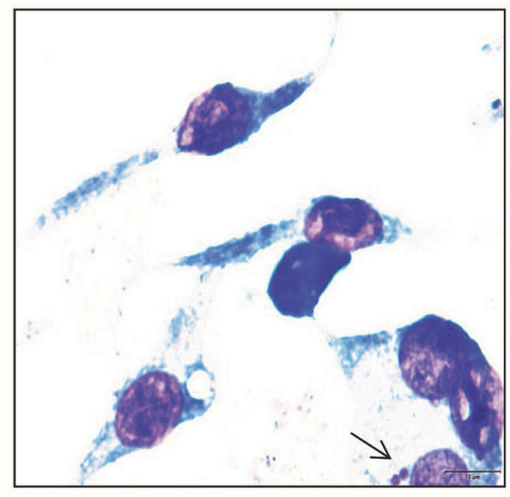

Cs-DSLN treated

Black arrows indicating L. donovani amastigotes

FIGURE 7 | (A) Percentage inhibition of Cs-SLN, AmBisome, and AmB against L. donovani-infected J774A.1 macrophages. (B) IC 50 values of Cs-SLN, AmBisome, and AmB against $L$. donovani-infected macrophages. (C) Microphotographs of Cs-SLN treated and untreated $L$. donovani-infected J774A.1 macrophages. Results are presented as mean \pm standard deviation $(n=3)$ and analyzed by Graph Pad Prism 8.0.2 software. (Cs-SLN and AmBisome) vs. AmB, ${ }^{\star \star} P<0.01,{ }^{\star} P<0.05$.

Moreover, chitosan being positively charged interact with cell membranes easily, possessing overall negative charge therefore expedites endocytosis (Singh et al., 2016b). Cs-SLN (1 $\mu \mathrm{g} / \mathrm{ml})$ showed a maximum percentage of inhibition (92.35\%) on intra-cellular amastigote growth of $L$. donovani (Figure 7A). Cs coating of SLN activates macrophages to release Th 1 cytokines which in turn generates an enhanced immunological response thereby facilitating the elimination of intracellular parasites (Jain et al., 2014) and AmB, a gold standard for leishmaniasis could be orally delivered by utilizing lipidbased nanocarriers with reduced adverse effect (Thanki et al., 2019).

\section{CONCLUSION}

Cs-SLN was successfully developed by the emulsion solvent evaporation method and well-characterized by FTIR, NMR, XRD, SEM, and TEM analysis. Cs-SLN has shown complete internalization in Caco-2 cells.
Cs-SLN has shown a sustained drug release profile over a period of $72 \mathrm{~h}$ and stable at gastric fluids, confirmed by simulated gastro-intestinal fluids study. Cs-SLN $(1 \mu \mathrm{g} / \mathrm{ml})$ have significantly $(P<0.01)$ reduced the intracellular amastigotes compared to free $\mathrm{AmB}$ without showing any toxic side effects. Additionally, surface modification could provide higher macrophage targeting and divulge an immunotherapeutic activity.

\section{DATA AVAILABILITY STATEMENT}

All datasets generated for this study are included in the article/supplementary material.

\section{AUTHOR CONTRIBUTIONS}

SP, GY, AK, OS, AV, SS, and SM: Methodology, Software, Data curation, Writing-Original draft preparation, Visualization, Investigation, Writing-Reviewing and Editing. SM: 
conceptualized the idea, acquired the funding, supervised the research work. All authors contributed to the article and approved the submitted version.

\section{FUNDING}

This work was supported by the Government of India for funding under DST-SERB Early Career Research Award $(\mathrm{ECR} / 2016 / 000977)$ and in part by the Department of

\section{REFERENCES}

Almeida, L. N. (2017). Solid lipid nanoparticles: the efficiency carrier for topical delivery of hydrophilic drugs. World J. Pharm. Pharm. Sci. 6, 175-189. doi: 10.20959/wjpps20179-10061

Bagde, A., Patel, K., Kutlehria, S., Chowdhury, N., and Singh, M. (2019). Formulation of topical ibuprofen solid lipid nanoparticle (SLN) gel using hot melt extrusion technique (HME) and determining its anti-inflammatory strength. Drug Deliv. Transl. Res. 9, 816-827. doi: 10.1007/s13346-019-00632-3

Battaglia, L., Gallarate, M., Cavalli, R., and Trotta, M. (2010). Solid lipid nanoparticles produced through a coacervation method. J. Microencapsul. 27, 78-85. doi: 10.3109/02652040903031279

Butani, D., Yewale, C., and Misra, A. (2016). Topical amphotericin B solid lipid nanoparticles: design and development. Colloids Surf. B. Biointerfaces 139, 17-24. doi: 10.1016/j.colsurfb.2015.07.032

Cavallaro, G., Craparo, E. F., and Sardo, C. (2015). PHEA-PLA biocompatible nanoparticles by technique of solvent evaporation from multiple emulsions. Int. J. Pharm. 495, 719-727. doi: 10.1016/j.ijpharm.2015.09.050

Channarong, S., Chaicumpa, W., Sinchaipanid, N., and Mitrevej, A. (2011). Development and evaluation of chitosan-coated liposomes for oral DNA vaccine: the improvement of Peyer's patch targeting using a polyplexloaded liposomes. AAPS PharmSciTech 12, 192-200. doi: 10.1208/s12249-0109559-9

Chappuis, F., Sundar, S., Hailu, A., Ghalib, H., Rijal, S., Peeling, R. W., et al. (2007). Visceral leishmaniasis: what are the needs for diagnosis, treatment and control? Nat. Rev. Microbiol. 5, 873-882. doi: 10.1038/nrmicro1748

Chavan, S. S., Ingle, S. G., and Vavia, P. R. (2013). Preparation and characterization of solid lipid nanoparticle-based nasal spray of budesonide. Drug Deliv. Transl. Res. 3, 402-408. doi: 10.1007/s13346-012-0105-z

Ching, M. S., Raymond, K., Bury, R. W., Mashford, M. L., and Morgan, D. J. (1983). Absorption of orally administered amphotericin B lozenges. Br. J. Clin. Pharmacol. 16, 106-108. doi: 10.1111/j.1365-2125.1983.tb02152.x

Costa, A., Sarmento, B., and Seabra, V. (2018). Mannose-functionalized solid lipid nanoparticles are e ff ective in targeting alveolar macrophages. Eur. J. Pharm. Sci. 114, 103-113. doi: 10.1016/j.ejps.2017.12.006

Farris, E., Brown, D. M., Ramer-Tait, A. E., and Pannier, A. K. (2017). Chitosan-zein nano-in-microparticles capable of mediating in vivo transgene expression following oral delivery. J. Control. Release 249, 150-161. doi: 10.1016/j.jconrel.2017.01.035

Ghadiri, M., Vatanara, A., Doroud, D., and Najafabadi, A. R. (2011). Paromomycin loaded solid lipid nanoparticles: characterization of production parameters. Biotechnol. Bioprocess Eng. 16, 617-623. doi: 10.1007/s12257-0100331-5

Guo, J., Ping, Q., Jiang, G., Huang, L., and Tong, Y. (2016). Chitosan-coated liposomes : characterization and interaction with leuprolide. Int. J. Pharm. 260, 167-173. doi: 10.1016/S0378-5173(03)00254-0

Guo, T., Zhang, Y., Zhao, J., Zhu, C., and Feng, N. (2015). Nanostructured lipid carriers for percutaneous administration of alkaloids isolated from Aconitum sinomontanum. J. Nanobiotechnol. 13:47. doi: 10.1186/s12951-015-0107-3

Gupta, S., Dube, A., and Vyas, S. P. (2012). Development and characterization of amphotericin B loaded solid lipid nanoparticles against experimental visceral leishmaniasis. Pharm. Nanotechnol. 1, 54-67. doi: $10.2174 / 2211738511301010054$
Science \& Technology (SR/NM/NS-57/2016), New Delhi (under Nano-Mission).

\section{ACKNOWLEDGMENTS}

SP and AK thank Institute of Nano Science and Technology for the Doctoral Fellowship. GY was thankful to the Department of Science \& Technology (DST) for providing financial support.

Guri, A., Gülseren, I., and Corredig, M. (2013). Utilization of solid lipid nanoparticles for enhanced delivery of curcumin in cocultures of HT29-MTX and Caco-2 cells. Food Funct. 4, 1410-1419. doi: 10.1039/c3fo60180c

Hasker, E., Singh, S. P., Malaviya, P., Picado, A., Gidwani, K., Singh, R. P., et al. (2012). Visceral leishmaniasis in rural Bihar, India. Emerg. Infect. Dis. 18, 1662-1664. doi: 10.3201/eid1810.111083

Herrera, G., Castillo, A., Ayala, M. S., Flórez, C., Cantillo-Barraza, O., and Ramirez, J. D. (2019). Evaluation of four rapid diagnostic tests for canine and human visceral Leishmaniasis in Colombia. BMC Infect. Dis. 19:747. doi: 10.1186/s12879-019-4353-0

Jain, S., Bhankur, N., Swarnakar, N. K., and Thanki, K. (2015). Phytantriol based "stealth" lyotropic liquid crystalline nanoparticles for improved antitumor efficacy and reduced toxicity of docetaxel. Pharm. Res. 32, 3282-3292. doi: 10.1007/s11095-015-1706-2

Jain, S., Siva, C., Reddy, K., Swami, R., and Kushwah, V. (2018). Amphotericin B loaded Chitosan nanoparticles : implication of bile salt stabilization on gastrointestinal stability, permeability and oral bioavailability. AAPS PharmSciTech 19, 3152-3164. doi: 10.1208/s12249-0181153-6

Jain, V., Gupta, A., Pawar, V. K., Asthana, S., Jaiswal, A. K., Dube, A., et al. (2014). Chitosan-assisted immunotherapy for intervention of experimental leishmaniasis via amphotericin B-loaded solid lipid nanoparticles. Appl. Biochem. Biotechnol. 174, 1309-1330. doi: 10.1007/s12010-0141084-y

Khan, W., and Kumar, N. (2011). Drug targeting to macrophages using paromomycin-loaded albumin microspheres for treatment of visceral leishmaniasis: an in vitro evaluation. J. Drug Target. 19, 239-250. doi: 10.3109/1061186X.2010.492524

Khatik, R., Dwivedi, P., Khare, P., Kansal, S., Dube, A., Mishra, P. R., et al. (2014). Development of targeted 1,2-diacyl-sn-glycero-3-phospho-1-serinecoated gelatin nanoparticles loaded with amphotericin B for improved in vitro and in vivo effect in leishmaniasis. Expert Opin. Drug Deliv. 11, 633-646. doi: $10.1517 / 17425247.2014 .889678$

Klein, S. (2010). The use of biorelevant dissolution media to forecast the in vivo performance of a drug. AAPS J. 12, 397-406. doi: 10.1208/s12248-010-9203-3

Kumar, P., and Bose, P. P. (2016). Targeted delivery of paromomycin to leishmania infected macrophage by hemoglobin tagged nanocarrier. J. Appl. Pharm. 8:212. doi: 10.4172/1920-4159.1000212

Kumar, S. R., Marianna, L., and Gianni, S. (2016). Synthesis and characterization of chitosan-coated magnetite nanoparticles and their application in curcumin drug delivery. Adv. Nat. Sci. Nanosci. Nanotechnol. 7:045010. doi: 10.1088/2043-6262/7/4/045010

Ling, J. T. S., Roberts, C. J., and Billa, N. (2019). Antifungal and mucoadhesive properties of an orally administered chitosan-coated amphotericin B nanostructured lipid carrier (NLC). AAPS PharmSciTech 20:136. doi: 10.1208/s12249-019-1346-7

Liu, D., Jiang, S., Shen, H., Qin, S., Liu, J., Zhang, Q., et al. (2011). Diclofenac sodium-loaded solid lipid nanoparticles prepared by emulsion/solvent evaporation method. J. Nanoparticle Res. 13, 2375-2386. doi: 10.1007/s11051-010-9998-y

Liu, M., Zhong, X., and Yang, Z. (2017). Chitosan functionalized nanocochleates for enhanced oral absorption of cyclosporine A. Sci. Rep. 7:41322. doi: $10.1038 /$ srep 41322 
Luo, Y., Zhang, B., Whent, M., Yu, L. L., and Wang, Q. (2011). Preparation and characterization of zein/chitosan complex for encapsulation of $\alpha$-tocopherol, and its in vitro controlled release study. Colloids Surf. B. Biointerfaces 85, 145-152. doi: 10.1016/j.colsurfb.2011.02.020

Ma, Z., and Lim, L. Y. (2003). Uptake of Chitosan and associated insulin in Caco-2 cell monolayers: a comparison between Chitosan molecules and Chitosan nanoparticles. Pharm. Res. 20, 1812-1819. doi: 10.1023/B:PHAM.0000003379.76417.3e

Martínez, C. R., and Ruiz, C. J. (2019). Alterations in host lipid metabolism produced during visceral leishmaniasis infections. Curr. Trop. Med. Rep. 6, 250-255. doi: 10.1007/s40475-019-00187-w

Mendonça, M. C. P., Radaic, A., Garcia-Fossa, F., da Cruz-Höfling, M. A., Vinolo, M. A. R., and de Jesus, M. B. (2020). The in vivo toxicological profile of cationic solid lipid nanoparticles. Drug Deliv. Transl. Res. 10, 34-42. doi: 10.1007/s13346-019-00657-8

Min, J. B., Kim, E. S., Lee, J. S., and Lee, H. G. (2018). Preparation, characterization, and cellular uptake of resveratrol-loaded trimethyl chitosan nanoparticles. Food Sci. Biotechnol. 27, 441-450. doi: 10.1007/s10068-0170272-2

Monge-Maillo, B., and López-Vélez, R. (2013). Therapeutic options for visceral leishmaniasis. Drugs 73, 1863-1888. doi: 10.1007/s40265-0130133-0

Mostafavi, M., Farajzadeh, S., Sharifi, I., Khazaeli, P., and Sharifi, H. (2019). Leishmanicidal effects of amphotericin B in combination with selenium loaded on niosome against Leishmania tropica. J. Parasit. Dis. 43, 176-185. doi: 10.1007/s12639-018-1071-2

Nahar, M., Mishra, D., Dubey, V., and Jain, N. K. (2009). "Development of amphotericin B loaded PLGA nanoparticles for effective treatment of visceral leishmaniasis," in IFMBE Proceedings (Singapore), 1241-1243. doi: 10.1007/978-3-540-92841-6_304

Nair, R., Kumar, A. C. K., Priya, V. K., Yadav, C. M., and Raju, P. Y. (2012). Formulation and evaluation of chitosan solid lipid nanoparticles of carbamazepine. Lipids Health Dis. 11:72. doi: 10.1186/1476-511X-11-72

Patra, J. K., Das, G., Fraceto, L. F., Campos, E. V. R., Rodriguez-Torres, M. D. P., Acosta-Torres, L. S., et al. (2018). Nano based drug delivery systems: recent developments and future prospects. J. Nanobiotechnol. 16, 1-33. doi: 10.1186/s12951-018-0392-8

Pauluk, D., Padilha, A. K., Khalil, N. M., and Mainardes, R. M. (2019). Chitosancoated zein nanoparticles for oral delivery of resveratrol: formation, characterization, stability, mucoadhesive properties and antioxidant activity. Food Hydrocoll. 94, 411-417. doi: 10.1016/j.foodhyd.2019. 03.042

Ponte-Sucre, A., Gamarro, F., Dujardin, J. C., Barrett, M. P., López-Vélez, R., García-Hernández, R., et al. (2017). Drug resistance and treatment failure in leishmaniasis: a 21st century challenge. PLoS Negl. Trop. Dis. 11:e0006052. doi: 10.1371/journal.pntd.0006052

Pozo-rodríguez, A., Solinís, M. A., Gascón, A. R., and Pedraz, J. L. (2009). Shortand long-term stability study of lyophilized solid lipid nanoparticles for gene therapy. Eur. J. Pharm. Biopharm. 71, 181-189. doi: 10.1016/j.ejpb.2008.09.015

Schipper, N. G., Olsson, S., Hoogstraate, J. A., deBoer, A. G., Vårum, K. M., and Artursson, P. (1997). Chitosans as absorption enhancers for poorly absorbable drugs 2: mechanism of absorption enhancement. Pharm. Res. 14, 923-929. doi: 10.1023/A:1012160102740
Shi, K., Xue, B., Jia, Y., Yuan, L., Han, R., Yang, F., et al. (2019). Sustained co-delivery of gemcitabine and cis-platinum via biodegradable thermosensitive hydrogel for synergistic combination therapy of pancreatic cancer. Nano Res. 12, 1389-1399. doi: 10.1007/s12274-019-2342-7

Shrestha, M., Pandey, B. D., Maharjan, J., Dumre, S. P., Tiwari, P. N., Manandhar, K., et al. (2018). Visceral leishmaniasis from a non-endemic Himalayan region of Nepal. Parasitol. Res. 117, 2323-2326. doi: 10.1007/s00436-0185887-6

Singh, O. P., Singh, B., Chakravarty, J., and Sundar, S. (2016a). Current challenges in treatment options for visceral leishmaniasis in India: a public health perspective. Infect. Dis. Poverty 5:19. doi: 10.1186/s40249-016-0112-2

Singh, P. K., Sah, P., Meher, J. G., Joshi, S., Pawar, V. K., Raval, K., et al. (2016b). Macrophage-targeted chitosan anchored PLGA nanoparticles bearing doxorubicin and amphotericin B against visceral leishmaniasis. RSC Adv. 6, 71705-71718. doi: 10.1039/C6RA06007B

Sundar, S., and Singh, A. (2016). Recent developments and future prospects in the treatment of visceral leishmaniasis. Ther. Adv. Infect. Dis. 3, 98-109. doi: $10.1177 / 2049936116646063$

Thanki, K., Date, T., and Jain, S. (2019). Improved oral bioavailability and gastrointestinal stability of amphotericin B through fatty acid conjugation approach. Mol. Pharm. 16, 4519-4529. doi: 10.1021/acs.molpharmaceut.9b00662

Trinconi, C. T., Reimão, J. Q., Yokoyama-Yasunaka, J. K. U., Miguel, D. C., and Uliana, S. R. B. (2014). Combination therapy with tamoxifen and amphotericin B in experimental cutaneous leishmaniasis. Antimicrob. Agents Chemother. 58, 2608-2613. doi: 10.1128/AAC.01315-13

Vijayakumar, A., Baskaran, R., Jang, Y. S., Oh, S. H., and Yoo, B. K. (2017). Quercetin-loaded solid lipid nanoparticle dispersion with improved physicochemical properties and cellular uptake. AAPS PharmSciTech 18, 875-883. doi: 10.1208/s12249-016-0573-4

Vivek, K., Reddy, H., and Murthy, R. S. R. (2007). Investigations of the effect of the lipid matrix on drug entrapment, in vitro release, and physical stability of olanzapine-loaded solid lipid nanoparticles. AAPS PharmSciTech 8, 16-24. doi: $10.1208 /$ pt0804083

Wang, J., Wang, Y., and Meng, X. (2016). Chitosan nanolayered Cisplatin-loaded lipid nanoparticles for enhanced anticancer efficacy in cervical cancer. Nanoscale Res. Lett. 11:524. doi: 10.1186/s11671-0161698-9

Yadagiri, G., and Singh, P. P. (2018). "Chemotherapy and experimental models of visceral leishmaniasis," in Infectious Diseases and Your Health, ed P. Singh (Singapore: Springer), 63-97. doi: 10.1007/978-981-13-1577-0_5

Conflict of Interest: The authors declare that the research was conducted in the absence of any commercial or financial relationships that could be construed as a potential conflict of interest.

Copyright (c) 2020 Parvez, Yadagiri, Karole, Singh, Verma, Sundar and Mudavath. This is an open-access article distributed under the terms of the Creative Commons Attribution License (CC BY). The use, distribution or reproduction in other forums is permitted, provided the original author $(s)$ and the copyright owner(s) are credited and that the original publication in this journal is cited, in accordance with accepted academic practice. No use, distribution or reproduction is permitted which does not comply with these terms. 